INPLASY

PROTOCOL

To cite: Yin et al. The efficacy of laparoscopic and roboticassisted cyst excision and hepaticojejunostomy for choledochal cyst: a systematic review and meta-analysis. Inplasy protocol 2021120024. doi:

10.37766/inplasy2021.12.0024

Received: 04 December 2021

Published: 04 December 2021

Corresponding author:

Tong Yin

719189245@qq.com

Author Affiliation:

Capital Institute of Pediatrics-

Peking University Teaching

Hospital, Beijing, China

Support: No financial support.

Review Stage at time of this submission: The review has not yet started.

Conflicts of interest:

None declared.

\section{The efficacy of laparoscopic and robotic- assisted cyst excision and hepaticojejunostomy for choledochal cyst: a systematic review and meta-analysis}

Yin, T1; Chen, SY2; Huang, $\mathrm{T}^{3}$.

Review question / Objective: The aim of this systematic review and meta-analysis is to evaluate the efficacy of laparoscopic and robotic-asissted cyst excision and hepaticojejunostomy for choledochal cysts.

Condition being studied: Choledochal cyst (CDC) is a congenital biliary dilation, with the higher risk in Eastern Asian and in female. As a precancerous lesion, operation is the only method for CDC patients. Traditionally, open surgery is predominant in surgeon. Farello first introduced laparoscopic operation for CDCs. Since then, the laparoscopic operation is being developed rapidly. In recent years, robotic-assisted operation was also applied for CDCs. But the efficacy of different methods has not been concluded.

INPLASY registration number: This protocol was registered with the International Platform of Registered Systematic Review and Meta-Analysis Protocols (INPLASY) on 04 December 2021 and was last updated on 04 December 2021 (registration number INPLASY2021120024).

\section{INTRODUCTION}

Review question / Objective: The aim of this systematic review and meta-analysis is to evaluate the efficacy of laparoscopic and robotic-asissted cyst excision and hepaticojejunostomy for choledochal cysts.
Condition being studied: Choledochal cyst (CDC) is a congenital biliary dilation, with the higher risk in Eastern Asian and in female. As a precancerous lesion, operation is the only method for CDC patients. Traditionally, open surgery is 
predominant in surgeon. Farello first introduced laparoscopic operation for CDCs. Since then, the laparoscopic operation is being developed rapidly. In recent years, robotic-assisted operation was also applied for CDCs. But the efficacy of different methods has not been concluded.

\section{METHODS}

Search strategy: We will search articles in six databases for relevant English language literature: PubMed, Embase, Web of Science, Cochrane Library, Clinical Trials and Wiley. The search string will be built as follows: (laparoscopic) AND (robotic) AND (choledochal cyst). The electronic database search will be supplemented by a manual search of the reference lists of included articles.

Participant or population: Accoding to our eligibility criteria, the choledochal cyst patients treated with laparoscopic or robotic-asisted operation were included.

Intervention: Whether to perform laparoscopic or robotic-assissted cyst excision depended on surgeron. If patients with bleeding, adhesion or other conditions that cannot finished by laparoscopy or robot, they would be converted to open operation.

Comparator: Laparoscopic operation demands one camera port in umbilicus and two or three trocar in right lower abdomen. In robotic-assissted operations, a $12 \mathrm{~mm}$ trocar was placed through the umbilicus as the camera port. Then the carbon dioxide pneumoperitoneum with a pressure of 8 to $12 \mathrm{mmHg}$ was established. Two $8 \mathrm{~mm} \mathrm{Da}$ Vinci trocars were placed on each side of the umbilicus. A $5 \mathrm{~mm}$ trocar was placed at the left lower abdomen as the assistant port. Both need free the gall bladder, ligate the cystic artery, excise the cyst, make the biliary-enteric Roux-loop, and make the hepatic-jejunum anastomsis.

Study designs to be included: Retrospective cochort study compared laparoscopic or robotic-assissted cyst excision and hepaticojejunostomy will be included.

Eligibility criteria: Inclusion criteria: (1) intervation: laparoscopic or roboticassissted cyst excision and hepaticojejunostomy (2) outcomes: study reported on at least one of the outcome measures mentioned below: operative time, blood loss, length of hospital stay, postoperative morbidity and mortality. Exclusion criteria: (1) review articles (2) meeting reports (3) studies only included one method (4) case reports (5) full text not in English.

Information sources: We will search articles in six databases for relevant English language literature: PubMed, Embase, Web of Science, Cochrane Library, Clinical Trials and Wiley. The search string will be built as follows: (laparoscopic) AND (robotic) AND (choledochal cyst). We will try to contact with authors. And we are registering our meta-analysis in INPLASY.

Main outcome(s): The primary outcomes were postoperative complications, for which would decrease the quality of life for patients significantly. We assessed all the complications reported by the included articles, including short-term (i.e. bile leakage, bleeding, pancreatic leakage, Roux-loop obstruction, adhesive intestinal obstruction, infection and etc.), long-term (i.e. anastomotic stenosis, cholelithiasis, cholangitis, malignancy and etc.), and total postoperative complication.

Additional outcome(s): Secondary outcomes were operational details (operative time, blood loss, conversion rate), and postoperative outcomes (hospital stays, duration of drainage, time to resume full diet) and so on.

Data management: Statistical analysis was conducted by Review Manager 5.4 version and Stata 15 version. Relative risk (RR) was applied for dichotomous variables and weighted mean differences (WMD) was applied for continuous variables. Some study outcomes were reported as medians with ranges or mid-quartile with ranges. 
According to the methods introduced by Wan and Hozo, those data were converted to means with deviations. The 12 statistic were used to test the degrees of heterogeneity. 12 greater than $50 \%$ was considered to be high heterogeneity and then the random-effects was applied to pool the results. However, 12 less than $50 \%$ was considered to be low heterogeneity, and then fixed-effects model was used.

Quality assessment / Risk of bias analysis: To assess the risk of bias, the "Risk of bias" assessment tool was applied on included records.

Strategy of data synthesis: Review manager is used to compare the continuous data, like operative time, blood loss, hostital stays, feeding time and duration of drainage, and dichotomous data, like postoperative complications. Stata is used to assess the publication bias of postoperative complications.To assess the risk of bias, the "Risk of bias" assessment tool was applied on included records.

Subgroup analysis: No subgroup analysis will be used in study.

Sensitivity analysis: To assess the effects of any single study, sensitivity analysis was conducted. The Grading of Recommendations Assessment, Development, and Evaluation (GRADE) approach is used to evaluate the quality of the evidence. The evidence was categorized as high, moderate, low or very low quality.

Language: English articles were recruited only.

Country(ies) involved: China.

Keywords: laparoscopic; robotic-assisted; choledochal cyst.

Contributions of each author:

Author 1 - Tong Yin.

Author 2 - Suyun Chen.

Author 3 - Ting Huang. 